Article

\title{
Enantioselective Synthesis of Homo- $N$-Nucleosides Containing a 1,4-Dioxane Sugar Analog
}

\section{Qiang Yu and Per Carlsen*}

Department of Chemistry, Norwegian University of Science and Technology (NTNU), N-7491

Trondheim, Norway; E-mail: qiang@nt.ntnu.no

* Author to whom correspondence should be addressed; E-mail: per.carlsen@chem.ntnu.no; Fax: +47 73594256.

Received: 3 September 2008; in revised form: 30 October 2088 / Accepted: 7 November 2008 / Published: 3 December 2008

\begin{abstract}
A dioxane homo-sugar analog, (2S,5S)-and (2R,5S)-5-[(4S)-2,2-dimethyl-1,3dioxolan-4-yl]-2-iodomethyl-1,4-dioxane was prepared from $(2 R, 3 R)$-dimethyl tartrate, and further elaborated into the corresponding homo- $N$-nucleoside analogs by its reactions with uracil and adenine, respectively.
\end{abstract}

Keywords: Sugar analog; Nucleoside analogs; Homo-N-Nucleoside; 1,4-Dioxane, Heterocycles.

\section{Introduction}

There has been an increasing interest in the synthesis of nucleoside analogs with modifications of the sugar moiety for the purpose of obtaining new antiviral and antitumor agents [1-3]. A well known class of modified nucleosides are the homo- $N$ - and $C$-glycosidic nucleosides [4-8] The insertion of a methylene group between the heterocyclic base and the sugar moiety results in a more flexible structure, and due to the lack of an anomeric acetal position, these nucleosides are in general resistant to enzymatic degradation [8]. For these reasons, we decided to pursue the synthesis of nucleoside analogs, however, based on the conformationally more flexible, optically active homo-1,4-dioxane sugar analogs. The objective was thus to construct novel homo- $N$-nucleoside analogs containing a 1,4- 
dioxane sugar moiety. A representative structure is shown in Figure 1, where the 1,4-dioxane homosugar analog is substituted with uracil or adenine, respectively.

Figure 1. A representative structure of the 1,4-dioxane homo- $N$-nucleoside analogs.

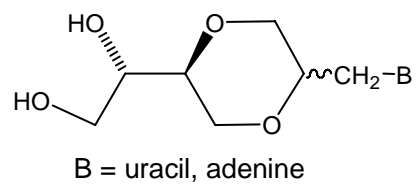

\section{Results and Discussion}

The formation of the 1,4-dioxane homo-sugar analog 4 was readily achieved starting from $(2 R, 3 R)$ dimethyl tartrate, an inexpensive and commercial available chiral starting material. Thus, $(2 R, 3 R)$ dimethyl tartrate was converted into the corresponding enantiomerically pure allyl ether $\mathbf{1}$ [9] either by the reaction with allyl bromide in the presence of silver oxide [10] or in a tin assisted reaction with dibutyltin oxide [11-12]. The dimethyl (2R,3R)-2-O-allyltartrate 1 was then reduced by $\mathrm{LiAlH}_{4}$ [13-15] or $\mathrm{NaBH}_{4}$ [16-17] to give triol 2. The two vicinal hydroxyl groups in 2 were next protected through formation of acetal 3 by the reaction with 2,2-dimethoxypropane in the presence of $p$-toluenesulfonic acid (Scheme 1). The use of the tartrates as chiral starting materials conveniently allows for the synthesis of all the possible, optically active stereoisomers of $\mathbf{3}$ and subsequently the corresponding homo-sugar analogs.

Scheme 1. Synthesis of the partially protected (2S,3S)-2(allyloxy)butane-1,3,4-triol, 3 .

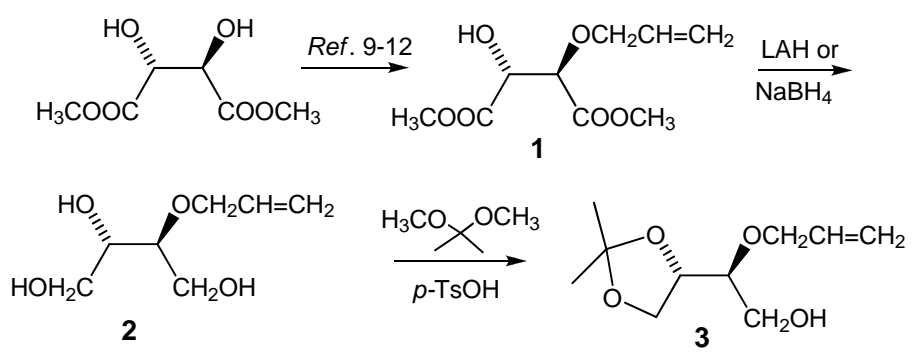

Iodocyclization of intermediate 3 in the presence of anhydrous $\mathrm{NaHCO}_{3}$ in dry acetonitrile [18-21] gave the 1,4-dioxane pseudo-sugar as a diastereomeric mixture of trans- and cis-iodides $\mathbf{4 a}$ and $\mathbf{4 b}$ in $26.4 \%$ and $25.4 \%$ isolated yields, respectively. The trans-compound $\mathbf{4 a}$ and cis-compound $\mathbf{4 b}$ were separated by flash chromatography using a solvent mixture of diethyl ether and $n$-hexane (gradient 1/4-1/1), Scheme 2.

Scheme 2. Formation of homo-sugar iodides $4 \mathbf{a}$ and $\mathbf{4 b .}$

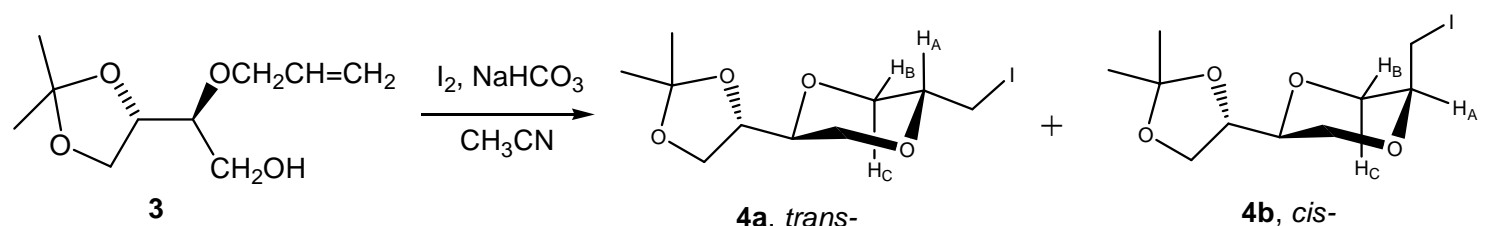


Structures $\mathbf{4 a}$ and $\mathbf{4 b}$ were elucidated and verified by ${ }^{1} \mathrm{H}-,{ }^{13} \mathrm{C}-$, and DEPT-NMR experiments in combination with 2D NMR spectroscopy techniques (COSY, HSQC, HMBC, NOESY). The assigned structures were in full agreement with the NMR data. Product 4a was assigned the trans-configuration as the coupling constants $J_{\mathrm{AC}}$ and $J_{\mathrm{AB}}$ were measured to $10.2 \mathrm{~Hz}$ and $2.4 \mathrm{~Hz}$, respectively. This was in agreement with the $-\mathrm{CH}_{2} \mathrm{I}$ group being in an equatorial position. The corresponding coupling values for the other isomer were $3.6 \mathrm{~Hz}$ and $3.3 \mathrm{~Hz}$, respectively, being in agreement with the structure of the cis-isomer $\mathbf{4 b}$.

The uracil homo- $N$-nucleoside analog 5 a was obtained by reacting uracil with sodium hydride in DMF [22], followed by the reaction with trans-iodide 4a. A byproduct containing two 1,4-dioxane rings was also obtained and assigned the structure 6a. This byproduct was not easily separated from product 5a. The acetal functions in compounds 5a and 6a were then removed using Amberlyst 15 in methanol providing a mixture of homo- $N$-nucleoside analog $7 \mathbf{a}$ and dimer $\mathbf{8 a}$, Scheme 3 . Compounds $\mathbf{7 a}$ and $\mathbf{8 a}$ were now readily separated by flash chromatography. The cis-nucleoside analogs $\mathbf{7 b}$ and $\mathbf{8 b}$ were obtained from $\mathbf{4 b}$ by the same sequence of reactions.

Scheme 3. Synthesis of uracil $N$-nucleoside analogs $7 \mathbf{a}$ and $\mathbf{7 b}$.

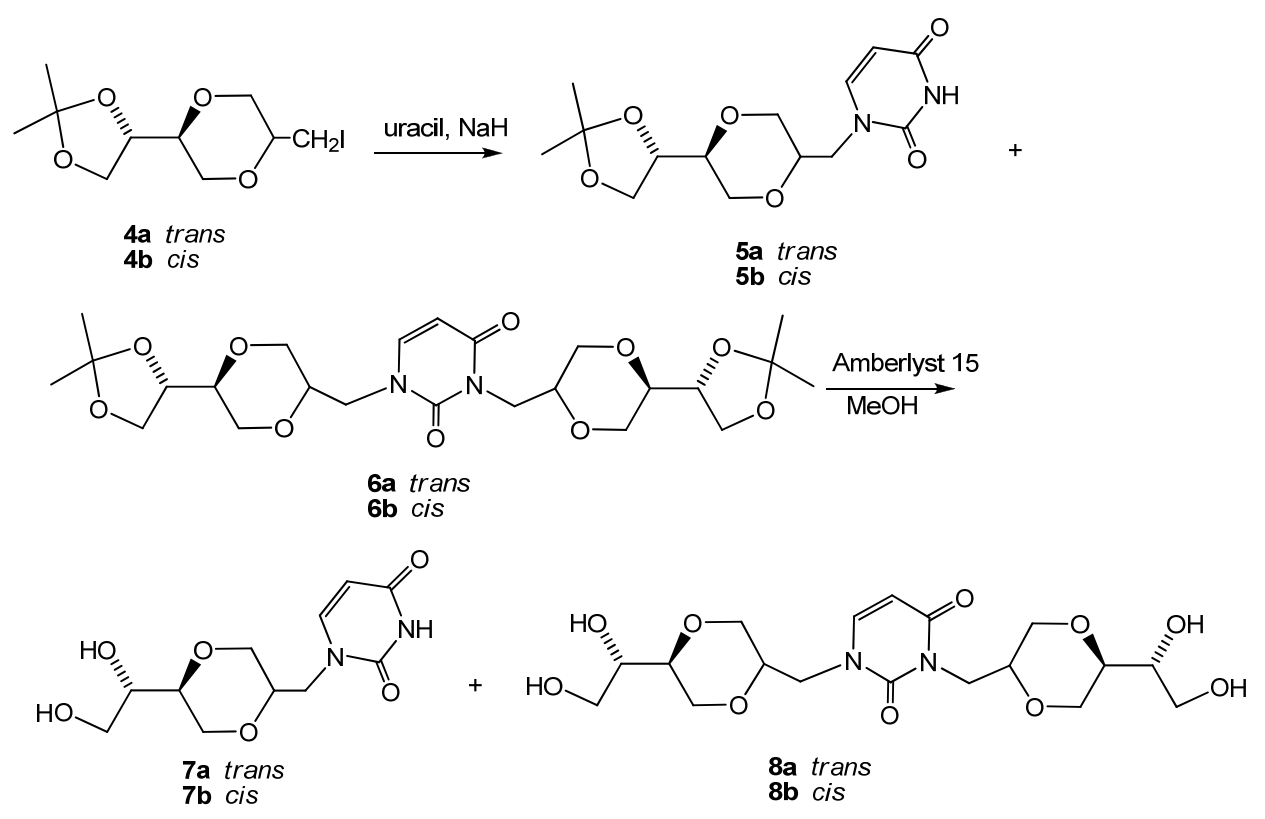

NMR spectra of the crude reaction mixtures gave indication of an additional byproduct, which as a working hypothesis was assumed to be the corresponding 3-regioisomer 10. To confirm the identity of the two regioisomers 5 and 10, uracil was first selectively protected as the $3-\mathrm{N}$ position by benzoylation with benzoyl chloride in pyridine to provide pure $N$-3-benzoyluracil 9, [23]. Compound 9 was next reacted with iodide $\mathbf{4 b}$ in DMF to give product $\mathbf{5 b}$ (Scheme 4). The NMR spectral data of $\mathbf{5 b}$ prepared by the two different routes were in good agreement. Interestingly, the N-3 alkylation compound $\mathbf{1 0}$ was also observed in the product from the protected uracil 9. A 6:5 ratio of products $\mathbf{5 b}$ and 10 was observed. The results imply that a benzoyl-walk reaction probably took place under the reaction conditions. The pure $\mathbf{1 0}$ was isolated from the mixture of $\mathbf{5 b}$ and $\mathbf{1 0}$ by preparative TLC and 
its structure was confirmed by NMR spectroscopy. The detailed nature of these transformations was not further investigated.

Scheme 4. Reaction of iodide $4 \mathbf{b}$ with 3-benzoyluracil, 9.
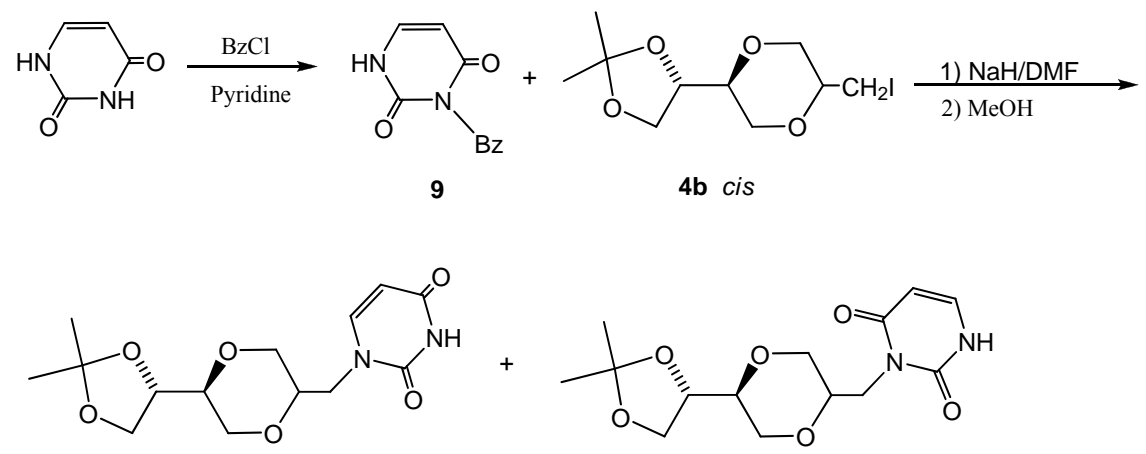

5b cis

10 cis

Using anhydrous potassium carbonate [24-25] as the base, the trans- iodide $\mathbf{4 a}$ and cis- iodide $\mathbf{4 b}$ respectively were reacted with adenine to give compound 11a and $\mathbf{1 1 b}$ in $42 \%$ and $30 \%$ isolated yields, respectively, after flash chromatography. Deprotection of acetals 11a and 11b in the presence of Amberlyst-15 in methanol gave compound 12a and 12b in $85 \%$ and $70 \%$ yields (Scheme 5). Different from natural occurring purine nucleosides, 12a and 12b were stable under acidic conditions. The depurination reaction was avoided due to the presence of the methylene group between adenine and 1,4-dioxane homo-sugar analog moiety.

Scheme 5. Synthesis of adenine $N$-nucleoside analogs 12a and $\mathbf{1 2 b}$.

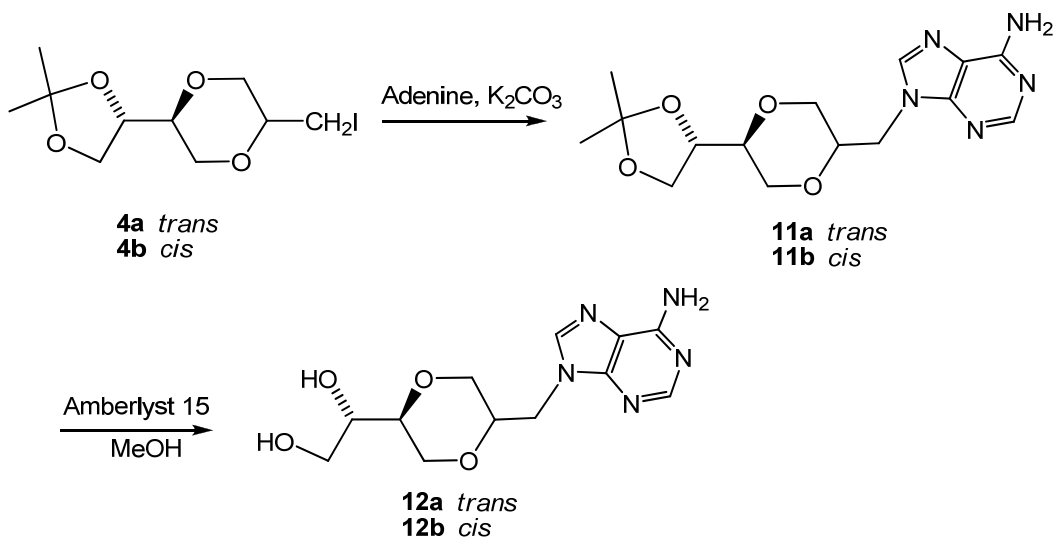

The structures of the four adenine nucleoside analogs 11a, 11b, 12a, 12b were verified to be the $N$ 9 adenine regioisomers by HMBC-NMR spectroscopy technique. In the case of 11a, three bond correlations, between $\mathrm{C} 4$ and $\mathrm{H}_{\mathrm{A}}$ and between $\mathrm{C} 8$ and $\mathrm{H}_{\mathrm{A}}$, were observed, while three bond correlation between $\mathrm{C} 5$ and $\mathrm{H}_{\mathrm{A}}$ was not found (Figure 2). These findings were in agreement with e.g. product 11a be the N-9 adenine regioisomer. 
Figure 2. Structure of adenine homo- $N$-nucleoside analog 11a.

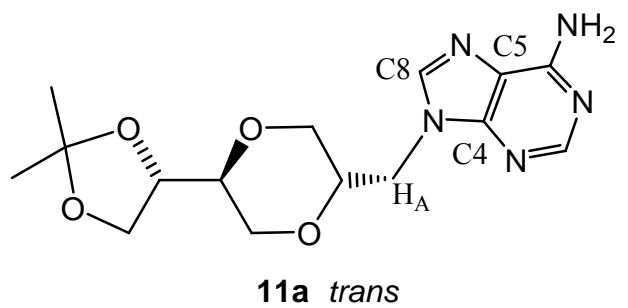

\section{Conclusions}

In conclusion, optically active homo- $N$-sugar nucleoside analogs containing a 1,4-dioxane moiety as the sugar analog and substituted with uracil or adenine as the base were synthesized from dimethyl tartrate. These nucleoside analogs were stable under acidic conditions. Plans for the biological screening of the produced nucleoside analogs are currently in progress.

\section{Experimental}

\section{General}

NMR spectra were recorded on Bruker Avance DPX 300 or DPX 400 instruments. Chemical shifts are reported in ppm using TMS as the internal standard in $\mathrm{CDCl}_{3}$ or relative to $2.50 \mathrm{ppm}$ for ${ }^{1} \mathrm{H}$ and $39.99 \mathrm{ppm}$ for ${ }^{13} \mathrm{C}$ in DMSO- $d_{6}$ or $3.31 \mathrm{ppm}$ for ${ }^{1} \mathrm{H}$ and $49.15 \mathrm{ppm}$ for ${ }^{13} \mathrm{C}$ in $\mathrm{CD}_{3} \mathrm{OD}$. Structural assignments were based on ${ }^{1} \mathrm{H},{ }^{13} \mathrm{C}$, DEPT135 and 2D spetra, COSY, HSQC, HMBC, NOESY. EIMass and ESI spectra were recorded on a Finnigan MAT 95XL spectrometer. IR spectra were obtained on a Thermo Nicolet FT-IR Nexus spectrometer using a Smart Endurance reflection cell. Silica gel Kieselgel 60G (Merck) was used for Flash Chromatography. The solvents were purified by standard methods. The preparations of compounds 1 were described elsewhere [9-12].

(2S,3S)-3-(allyloxy)butane-1,2,4-triol, (2)

This product was obtained by reduction of 1 with either $\mathrm{LiAlH}_{4}$ or $\mathrm{NaBH}_{4} \cdot \mathrm{LiAlH}_{4}$ reduction: To a suspension of $\mathrm{LiAlH}_{4}(3.72 \mathrm{~g}, 95 \%, 93 \mathrm{mmol})$ in dry diethyl ether $(50 \mathrm{~mL})$ was drop wise added a solution of 1 ( $4.36 \mathrm{~g}, 20 \mathrm{mmol})$ in $4 \mathrm{~mL}$ of diethyl ether at $0-5{ }^{\circ} \mathrm{C}$. The reaction mixture was refluxed for 18 hours and then cooled in an ice bath. Then $5 \mathrm{~mL}$ of water was added and the mixture stirred for 20 minutes, followed by addition of a $15 \% \mathrm{NaOH}$ solution $(12 \mathrm{~mL})$ and then $10 \mathrm{~mL}$ of water. The resulting mixture was stirred and the granular salt formed, was separated by filtration, washed with hot THF (200 mL), and the filtrate concentrated under reduced pressure. The residue was purified by flash chromatography $\left(\mathrm{CHCl}_{3} / \mathrm{CH}_{3} \mathrm{OH}, 9: 1\right.$ mixture $)$ to give $0.83 \mathrm{~g}, 26 \%$ of the pure product 2 . $\mathrm{NaBH}_{4}$ reduction. Sodium borohydride $(3.45 \mathrm{~g}, 93 \mathrm{mmol})$ in ethanol $(50 \mathrm{~mL})$ was stirred for half an hour and then dropwise added a solution of $1(4.35 \mathrm{~g}, 20 \mathrm{mmol})$ in ethanol $(15 \mathrm{~mL})$. The resulting solution was refluxed gently for 5 hours. The solution was cooled in an ice bath and added $10 \mathrm{~mL}$ of acetic acid. The mixture was stirred for 20 minutes and filtered. The solid was washed with $2 \times 50 \mathrm{ml}$ ethanol. The 
combined organic phase was concentrated under reduced pressure. The crude product was purified by flash chromatography using a 19:1 mixture of $\mathrm{CH}_{2} \mathrm{Cl}_{2} / \mathrm{MeOH}$ as the eluent yielding $2.88 \mathrm{~g}, 88 \%$ of the pure product, which exhibited the following spectroscopic properties: ${ }^{1} \mathrm{H}-\mathrm{NMR}\left(\mathrm{CDCl}_{3}, 400\right.$ $\mathrm{MHz}$ ): $\delta=3.45$ (q, $1 \mathrm{H}, \mathrm{CH}-\mathrm{OAllyl}$ ), 3.66-3.74 (m, 3H, $1 \mathrm{H}$ from $\mathrm{CH}_{2}-\mathrm{CHOH}, 2 \mathrm{H}$ from $\mathrm{CH}_{2}-\mathrm{CH}-\mathrm{OAll}$ ), 3.80 (dd, $1 \mathrm{H}$ from $\left.\mathrm{CH}_{2}-\mathrm{CHOH}\right), 3.86(\mathrm{q}, 1 \mathrm{H}, \mathrm{CHOH}), 4.03-4.20\left(\mathrm{~m}, 2 \mathrm{H}, \mathrm{OCH}_{2}-\mathrm{CH}=\mathrm{CH}_{2}\right), 4.32$ (s, broad, $3 \mathrm{H}, \mathrm{OH}), 5.18-5.38\left(\mathrm{~m}, 2 \mathrm{H}, \mathrm{CH}_{2}=\mathrm{CH}\right), 5.86-5.96\left(\mathrm{~m}, 1 \mathrm{H}, \mathrm{CH}=\mathrm{CH}_{2}\right)$ ppm. ${ }^{13} \mathrm{C}-\mathrm{NMR}\left(\mathrm{CDCl}_{3}\right.$, 100MHz): $\delta=60.6,63.3,71.6,71.8,79.1,117.8,134.5$ ppm. MS (EI) m/z: $145\left(\mathrm{M}^{+}-\mathrm{OH}\right), 131\left(\mathrm{M}^{+}-\right.$ $\left.\mathrm{CH}_{2} \mathrm{OH}\right), 101\left(\mathrm{OH}-\mathrm{CH}_{2}=\mathrm{O}^{+} \mathrm{CH}_{2}-\mathrm{CH}=\mathrm{CH}_{2}\right), 61\left(\mathrm{HOCH}_{2} \mathrm{CH}=\mathrm{O}^{+} \mathrm{H}\right)$. IR (neat): 3365, 2881, 1736, 1448 $\mathrm{cm}^{-1}$.

(S)-2-(allyloxy)-2-((S)-2,2-dimethyl-1,3-dioxolan-4-yl)ethanol

A solution of 2 (6.20 g, $38 \mathrm{mmol})$, 2,2-dimethoxylpropane (4.00 g, $38.5 \mathrm{mmol})$ and $p$-TsOH (223 $\mathrm{mg}, 1.2 \mathrm{mmol}$ ) in $100 \mathrm{~mL}$ acetone was stirred overnight at room temperature. The solvent was then removed and the residue was purified by flash chromatography using a 3:2 mixture of $\mathrm{Et}_{2} \mathrm{O} / \mathrm{n}$-hexane as the eluent to provide product 3 as acolorless oil (5.11 g, 85\%). Unreacted starting material 2 (1.05 g crude product) was recovered by continued elusion with a 19:1 mixture of $\mathrm{CH}_{2} \mathrm{Cl}_{2} / \mathrm{MeOH}$. Product 3 exhibited the following spectroscopic properties: ${ }^{1} \mathrm{H}-\mathrm{NMR}\left(\mathrm{CDCl}_{3}, 400 \mathrm{MHz}\right): \delta=1.37\left(\mathrm{~s}, 3 \mathrm{H}, \mathrm{CH}_{3}\right)$, 1.44 (s, $\left.1 \mathrm{H}, \mathrm{CH}_{3}\right), 2.48$ (s, broad, $\left.1 \mathrm{H}, \mathrm{OH}\right), 3.49-3.53$ (m, 1H, CH-OAll), 3.59 (dd, J=10.8Hz, 12Hz, $\left.1 \mathrm{H}, \mathrm{HOCH}_{2}-\mathrm{CHOAll}\right), 3.73\left(\mathrm{dd}, J=4.2 \mathrm{~Hz}, 12 \mathrm{~Hz}, 1 \mathrm{H}, \mathrm{HOCH}_{2}-\mathrm{CHOAll}\right), 3.81$ (dd, $J=7.2 \mathrm{~Hz}, 8.4 \mathrm{~Hz}$, $\left.1 \mathrm{H}, \mathrm{C}-\mathrm{OCH}_{2} \mathrm{CHO}-\mathrm{C}\right), 4.03\left(\mathrm{dd}, J=6.4 \mathrm{~Hz}, 8.4 \mathrm{~Hz}, 1 \mathrm{H}, \mathrm{C}-\mathrm{OCH}_{2} \mathrm{CHO}-\mathrm{C}\right), 4.18-4.22$ (m, $2 \mathrm{H}, \mathrm{OCH}_{2}-$ $\left.\mathrm{CH}=\mathrm{CH}_{2}\right), 4.26-4.31\left(\mathrm{~m}, 1 \mathrm{H}, \mathrm{C}-\mathrm{OCH}_{2} \mathrm{CHO}-\mathrm{C}\right), 5.24-5.33\left(\mathrm{~m}, 2 \mathrm{H}, \mathrm{CH}_{2}=\mathrm{CH}\right), 5.88-5.95(\mathrm{~m}, 1 \mathrm{H}$, $\left.\mathrm{CH}=\mathrm{CH}_{2}\right) \mathrm{ppm} .{ }^{13} \mathrm{C}-\mathrm{NMR}\left(\mathrm{CDCl}_{3}, 100 \mathrm{MHz}\right): \delta 25.3,26.4,61.6,65.4,71.8,76.4,79.1,109.4,117.4$, 134.7 ppm. MS: (EI) m/z: 202( $\left.\mathrm{M}^{+}\right), 187\left(\mathrm{M}^{+}-\mathrm{CH}_{3}\right), 171\left(\mathrm{M}^{+}-\mathrm{CH}_{2} \mathrm{OH}\right), 101\left(\mathrm{C}_{5} \mathrm{H}_{9} \mathrm{O}_{2}{ }^{+}\right)$.

(2S,5S)-5-[(4S)-2,2-dimethyl-1,3-dioxolan-4-yl]-2-iodomethyl-1,4-dioxane (4a) and (2R,5S)-5-[(4S)2,2-dimethyl-1,3-dioxolan-4-yl]-2-iodomethyl-1,4-dioxane (4b)

To a solution of $3(3.20 \mathrm{~g}, 15.8 \mathrm{mmol})$ in dry acetonitrile $(50 \mathrm{~mL})$ was added $\mathrm{NaHCO}_{3}(4.19 \mathrm{~g}, 49.9$ $\mathrm{mmol})$ at $-15^{\circ} \mathrm{C}$. The mixture was stirred for 10 minutes and iodine $(12.10 \mathrm{~g}, 47.7 \mathrm{mmol})$ was added. The reaction mixture was stirred for 68 hours with exclusion of light at -15 to $-0^{\circ} \mathrm{C}$. Ethyl acetate $(80$ $\mathrm{mL}$ ) was added to the mixture and the solution was neutralized by saturated sodium thiosulfate solution until a colorless solution was obtained. The aqueous phase was extracted with ethyl acetate and the combined organic phase was dried over anhydrous sodium sulfate. The solution was filtered and evaporated. The residue was purified by gradient column chromatography using $\mathrm{Et}_{2} \mathrm{O} / n$-hexane $(1: 4,1: 1)$ as eluent. The two diastereomers were separated in yields of $26.4 \%$ (4a) and $25.4 \%$ (4b). The pure compounds were white solid. $\mathrm{R}_{\mathrm{f}}$ was 0.43 and 0.36 respectively ( $n$-hexane/Et $2 \mathrm{O} 1: 1$ ). The product 4a exhibited the following spectroscopic properties: ${ }^{1} \mathrm{H}-\mathrm{NMR}\left(\mathrm{CDCl}_{3}, 400 \mathrm{MHz}\right) \delta=4.10$ 4.02 (m, 1H, H-4”), 4.04 (dd, 1H, J=11.6 Hz, 2.4 Hz, H-3 eq), 3.97 (dd, 1H, J= 8.0 Hz, 6.6 Hz, H-5”), 3.79 (dd, 1H, J=8.0 Hz, 6.8 Hz, H-5”), 3.79-3.53 (m, 4H, H-2, H-5', H-6), 3.39 (dd, 1H, J= 11.6 Hz, $\left.10.2 \mathrm{~Hz}, \mathrm{H}-3_{\mathrm{ax}}\right), 3.07$ (d, $\left.2 \mathrm{H}, J=6.0 \mathrm{~Hz}, \mathrm{H}-7\right), 1.42$ (d, $\left.3 \mathrm{H},{ }^{5} J_{\mathrm{H}-4}{ }^{\prime}-\mathrm{H}-8=0.4 \mathrm{~Hz}, \mathrm{H}-8\right), 1.35\left(\mathrm{~d}, 3 \mathrm{H},{ }^{5} J_{\mathrm{H}-}\right.$ 
$4 "-\mathrm{H}-8=0.4 \mathrm{~Hz}, \mathrm{H}-8) \mathrm{ppm} ;{ }^{13} \mathrm{C}-\mathrm{NMR}\left(\mathrm{CDCl}_{3}, 100 \mathrm{MHz}\right) \delta=109.9,75.4,75.0,74.2,70.9,67.9,65.3$, 26.4, 25.5, 25.4 ppm; HRMS (ESI) m/z: for $\mathrm{C}_{10} \mathrm{H}_{17} \mathrm{IO}_{4}[\mathrm{M}+\mathrm{Na}]^{+}$, Calcd. 351.0069, Found 351.0063. 4b: ${ }^{1} \mathrm{H}-\mathrm{NMR}\left(\mathrm{CDCl}_{3}, 400 \mathrm{MHz}\right): \delta=4.29(\mathrm{dd}, 1 \mathrm{H}, J=6.8 \mathrm{~Hz}, 13.2 \mathrm{~Hz}, \mathrm{H}-4 ”), 4.02$ (dd, $1 \mathrm{H}, J=8.0$ Hz, 6.4 Hz, H-5”), 3.97 (dd, 1H, J = 12.0 Hz, 3.6 Hz, H-3eq), 3.84 (dd, 1H, J=12.0 Hz, 3.0 Hz, H3ax), 3.80-3.75 (m, $1 \mathrm{H}, \mathrm{H}-2), 3.75$ (dd, $1 \mathrm{H}, J=8.0 \mathrm{~Hz}, J=6.8 \mathrm{~Hz}, \mathrm{H}-5$ "), 3.67 (dd, 1H, $J=12.4 \mathrm{~Hz}$, $\left.8.0 \mathrm{~Hz}, \mathrm{H}-6_{\mathrm{ax}}\right), 3.61-3.57$ (m, $\left.1 \mathrm{H}, \mathrm{H}-5\right), 3.60$ (dd, 1H, J = 12.4 Hz, $\left.2.8 \mathrm{~Hz}, \mathrm{H}-6_{\mathrm{eq}}\right), 3.42$ (dd, $1 \mathrm{H}, J=7.0$ $\mathrm{Hz}, 12.6 \mathrm{~Hz}, \mathrm{H}-7 \mathrm{a}), 3.40$ (dd, $1 \mathrm{H}, J=7.0 \mathrm{~Hz}, 13.0 \mathrm{~Hz}, \mathrm{H}-7 \mathrm{~b}), 1.44$ (d, $\left.3 \mathrm{H},{ }^{5} J_{\mathrm{H}-4 \text { " }-\mathrm{H}-8}=0.4 \mathrm{~Hz}, \mathrm{H}-8\right)$, $1.38\left(\mathrm{~d}, 3 \mathrm{H},{ }^{5} J_{\mathrm{H}-4}{ }^{\prime \prime-H}-8=0.4 \mathrm{~Hz}, \mathrm{H}-8\right) \mathrm{ppm} ;{ }^{13} \mathrm{C}-\mathrm{NMR}\left(\mathrm{CDCl}_{3}, 100 \mathrm{MHz}\right) \delta=109.8,74.8,73.4,72.5$, 66.3, 65.5, 62.3, 26.4, 25.3, 3.2 ppm; HRMS (ESI) m/z: for $\mathrm{C}_{10} \mathrm{H}_{17} \mathrm{IO}_{4}[\mathrm{M}+\mathrm{Na}]^{+}$, Calcd. 351.0069, Found 351.0079; IR (neat): 2980, 2867, 1461, 1413, 1380, $1370 \mathrm{~cm}^{-1}$.

Figure 3. Structures $\mathbf{4 a}$ and $\mathbf{4 b}$.
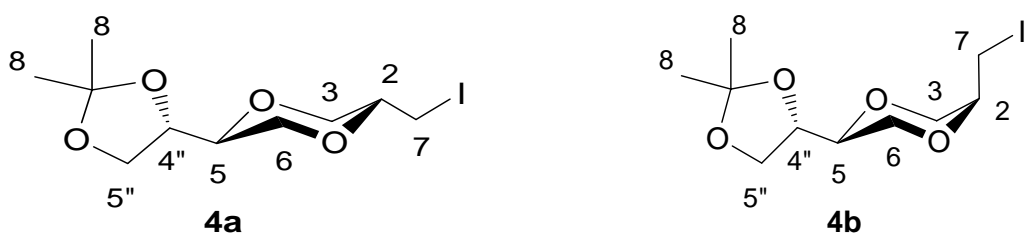

(2S,5S)-5-[(4S)-2,2-dimethyl-1,3-dioxolan-4-yl]-2-(uracil-1-yl-methyl)-1,4-dioxane (5a)

To a stirred suspension of uracil $(0.253 \mathrm{~g}, 2.3 \mathrm{mmol})$ in dry DMF $(19 \mathrm{~mL})$, sodium hydride $(0.065$ $\mathrm{g}, 2.7 \mathrm{mmol})$ was added at room temperature. After stirring for one hour, compound $\mathbf{4 a}(0.35 \mathrm{~g}, 1.1$ mmol) was added. The mixture was heated to $80^{\circ} \mathrm{C}$ and stirred overnight. The resulting mixture was evaporated under high vacuum. The residue was extracted with ethyl acetate. The solution was concentrated and purified by flash chromatography using ethyl acetate as the eluent. The product (201 $\mathrm{mg}$ ) containing the inseparable byproduct $\mathbf{6 a}$ was obtained in $60 \%$ crude yield. The product $5 \mathbf{a}$ exhibited the following spectroscopic properties: ${ }^{13} \mathrm{C}-\mathrm{NMR}\left(\mathrm{CDCl}_{3}, 100 \mathrm{MHz}\right): \delta=163.3,150.9$, 145.6, 109.8, 101.8, 75.2, 74.9, 73.3, 68.3, 67.4, 65.0, 48.8, 26.3, $25.2 \mathrm{ppm}$. The product contains inseparable byproduct 6a which makes the assignments of protons difficult; IR (neat) of the mixture of 5a and 6a: 3214, 3093, 2983, 2869, 1659, 1453, $1054 \mathrm{~cm}^{-1}$; HRMS (ESI) m/z: for $\mathrm{C}_{14} \mathrm{H}_{20} \mathrm{~N}_{2} \mathrm{O}_{6}$ $[\mathrm{M}+\mathrm{Na}]^{+}$, Calcd. 335.1219, Found 335.1222.

Figure 4. Structure 5a.

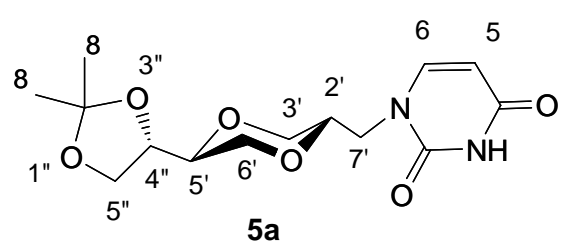


(2R,5S)-5-[(4S)-2,2-dimethyl-1,3-dioxolan-4-yl]-2-(uracil-1-yl-methyl)-1,4-dioxane (5b)

Method 1: The preparation of $\mathbf{5 b}$ was the same as used for the synthesis of 5a. Method 2: Sodium hydride (18 mg, $0.75 \mathrm{mmol}$ ) in $10 \mathrm{~mL}$ of dry DMF was stirred for half an hour at room temperature. $N$ 3-benzoyluracil 9 (130 mg, $0.60 \mathrm{mmol}$ ) was added and stirred for an hour. To this suspension, iodide 4b $(98 \mathrm{mg}, 0.30 \mathrm{mmol})$ was added. The resulting stirred mixture was heated at $90^{\circ} \mathrm{C}$ overnight. The mixture was concentrated under reduced pressure to remove DMF. To the residue was added $20 \mathrm{~mL}$ methanol and the resulting mixture was stirred for 5 minutes. The solution was concentrated and purified by flash chromatography using $\mathrm{CH}_{2} \mathrm{Cl}_{2} / \mathrm{CH}_{3} \mathrm{OH}$ as the eluent. The obtained product (30 mg, $32 \%$ ) containing products $\mathbf{5 b}$ and $\mathbf{1 0}$ in a 5:6 ratio and was further purified by preparative TLC. The isolated product $\mathbf{5 b}$ exhibited the following spectroscopic properties: ${ }^{1} \mathrm{H}-\mathrm{NMR}\left(\mathrm{CDCl}_{3}, 400 \mathrm{MHz}\right): \delta=$ 1.39, 1.45 (s, 2x3H, H8), 3.60 (m, 1H, H-5'), 3.67-3.74 (m, 2H, H-6'), 3.74-3.80 (m, 2H, H-5" and H3'), 3.82-3.86 (m, 1H, H-3'), 3.91-3.96 (m, 3H, H-7' and H-2'), 4.05 (dd, 1H, J=8.2 Hz, 6.6 Hz, H5”), 4.32-4.37 (m, 1H, H-4”), 5.70 (d, 1H, J=7.8 Hz, H-5), 7.20 (d, 1H, J=7.8 Hz, H-6), 8.73 (brs, 1H, $\mathrm{NH}) \mathrm{ppm} ;{ }^{13} \mathrm{C}-\mathrm{NMR}\left(\mathrm{CDCl}_{3}, 100 \mathrm{MHz}\right): \delta=163.3,150.8,145.1,109.9,102.1,74.4,73.8,71.0,65.7$, 64.9, 63.3, 47.6, 26.5, 25.4 ppm; IR (neat): 3097, 2985, 2874, 1682, 1652, 1455, 1124, 1060; HRMS (ESI) $\mathrm{m} / \mathrm{z}$ : for $\mathrm{C}_{14} \mathrm{H}_{20} \mathrm{~N}_{2} \mathrm{O}_{6}[\mathrm{M}+\mathrm{Na}]^{+}$, Calcd. 335.1219, Found 335.1222. The product 10 exhibited the following spectroscopic properties: ${ }^{1} \mathrm{H}-\mathrm{NMR}\left(\mathrm{CDCl}_{3}, 400 \mathrm{MHz}\right): \delta=1.38,1.45\left(2 \times 3 \mathrm{H}, \mathrm{CH}_{3}\right), 3.41$ (dd, 1H, J = 4 Hz, $12 \mathrm{~Hz}), 3.62-3.67(\mathrm{~m}, 1 \mathrm{H}), 3.70-3.75(\mathrm{~m}, 2 \mathrm{H}), 3.87$ (d, 2H), 3.97-4.08 (m, 3H),

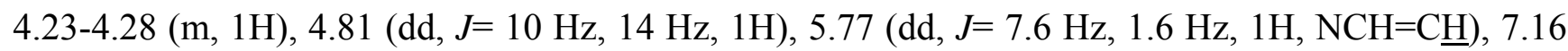
$(\mathrm{dd}, J=7.6 \mathrm{~Hz}, 5.6 \mathrm{~Hz}, 1 \mathrm{H}, \mathrm{NCH}=\mathrm{CH}), 8.66(\mathrm{~d}, 1 \mathrm{H}) \mathrm{ppm} ;{ }^{13} \mathrm{C}-\mathrm{NMR}\left(\mathrm{CDCl}_{3}, 100 \mathrm{MHz}\right): \delta=25.3,26.4$, $39.8,61.7,65.4,66.9,69.1,74.8,76.0,102.2,109.6,137.8,151.8,163.0 \mathrm{ppm} ; \mathrm{MS}(\mathrm{m} / \mathrm{z}):(\mathrm{M}+\mathrm{Na})^{+}$, 335.16 .

Figure 5. Structures $5 \mathbf{b}$ and 10.
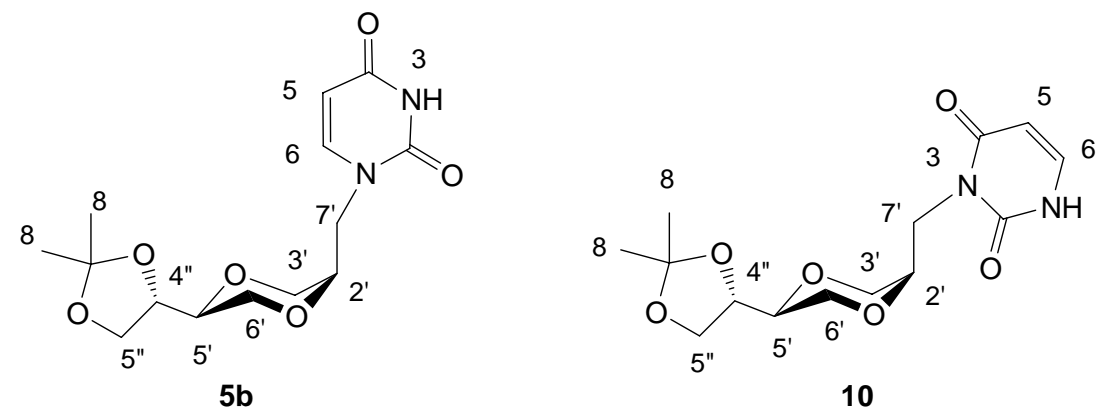

(2S,5S)-5-[(1S)-1, 2-dihydroxyethyl]-2-(uracil-1-yl-methyl)-1,4-dioxane (7a)

The mixture of $5 \mathbf{a}$ and $\mathbf{6 a}(60 \mathrm{mg}, 5 / 6$ ratio) was dissolved in methanol $(10 \mathrm{~mL})$, Amberlyst 15 (50 mg) was added and the mixture refluxed. The reaction was monitored by TLC until no more 7a was observed. The solution was filtered and the solvent was evaporated. The obtained product was further purified by flash chromatography to give pure 5a (12 mg, $34 \%)$ and 8 a $(25 \mathrm{mg}, 38 \%)$ using $\mathrm{CH}_{2} \mathrm{Cl}_{2} / \mathrm{MeOH}(13: 1)$ as eluent. The product 7a exhibited the following spectroscopic properties: ${ }^{1} \mathrm{H}-$ NMR ( $\left.\mathrm{CD}_{3} \mathrm{OD}, 400 \mathrm{MHz}\right): \delta=3.32-3.38$ (m, 1H, H-3'), 3.47-3.55 (m, 2H, H-1" and H-2"), 3.55-3.67 (m, 4H, H-5', H-2', H-6' and H-7'), 3.74-3.80 (m, 1H, H-2'), 3.82 (dd, 1H, J=1.2 Hz, 10.4 Hz, H-6'), 
3.88 (dd, 1H, J=2.8 Hz, 8.4 Hz, H-3'), 3.91 (dd, 1H, J=3 Hz, $11 \mathrm{~Hz}, \mathrm{H}-7$ '), 5.61 (d, 1H, J= $8 \mathrm{~Hz}, \mathrm{H}-$ 5), 7.52 (d, $1 \mathrm{H}, J=8 \mathrm{~Hz}, \mathrm{H}-6) \mathrm{ppm} ;{ }^{13} \mathrm{C}-\mathrm{NMR}\left(\mathrm{CD}_{3} \mathrm{OD}, 100 \mathrm{MHz}\right): \delta=50.0,63.7,69.3,69.7,72.6$, 74.4, 76.6, 101.8, 148.5, 153.0, 166.9 ppm; IR (neat): 3396, 2871, 1651, 1455, 1101, $1043 \mathrm{~cm}^{-1}$; HRMS (ESI) m/z: for $\mathrm{C}_{11} \mathrm{H}_{16} \mathrm{~N}_{2} \mathrm{O}_{6}[\mathrm{M}+\mathrm{Na}]^{+}$, Calcd. 295.0906, Found 295.0911.

Figure 6. Structure 7a.

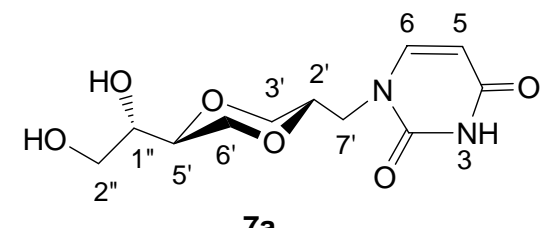

The product 8a exhibited the following spectroscopic properties: ${ }^{1} \mathrm{H}-\mathrm{NMR}\left(\mathrm{CD}_{3} \mathrm{OD}, 400 \mathrm{MHz}\right): \delta=$ 3.34-3.43 (m, 2H), 3.46-3.54 (m, 4H), 3.57-3.74 (m, 8H), 3.75-3.86 (m, 6H), 3.87-3.90 (m, 1H), 3.92$3.96(\mathrm{~m}, 1 \mathrm{H}), 4.05-4.10(\mathrm{~m}, 1 \mathrm{H}), 5.70(\mathrm{~d}, 1 \mathrm{H}, J=7.8 \mathrm{~Hz}, \mathrm{NCH}=\mathrm{CH}), 7.52(\mathrm{~d}, 1 \mathrm{H}, J=7.8 \mathrm{~Hz}$, $\mathrm{NCH}=\mathrm{CH}) \mathrm{ppm} ;{ }^{13} \mathrm{C}-\mathrm{NMR}\left(\mathrm{CD}_{3} \mathrm{OD}, 100 \mathrm{MHz}\right): \delta=42.8,51.1,63.7,63.8,69.3,69.4,69.7,72.57$, 72.61, 74.0, 74.4, 76.56, 76.57, 101.2, 146.8, 153.3, 165.6 ppm; MS (m/z): HRMS (ESI) m/z: for $\mathrm{C}_{18} \mathrm{H}_{28} \mathrm{~N}_{2} \mathrm{O}_{10}[\mathrm{M}+\mathrm{Na}]^{+}$, Calcd. 455.1641, Found 455.1645.

(2R,5S)-5-[(1S)-1,2-dihydroxyethyl]-2-(-uracil-1-yl-methyl)-1,4-dioxane (7b)

The method for preparation of $\mathbf{7 b}$ was as same as applied for the synthesis of $\mathbf{7 a}$. The product $\mathbf{7 b}$ exhibited the following spectroscopic properties: ${ }^{1} \mathrm{H}-\mathrm{NMR}\left(\mathrm{CD}_{3} \mathrm{OD}, 400 \mathrm{MHz}\right): \delta=3.53-3.66(\mathrm{~m}, 3 \mathrm{H}$, H-2" and H-6'), 3.60-3.70 (m, 2H, H-1' and H-5'), 3.78-3.80 (m, 2H, H-3'), 3.80-3.92 (2H, H-2' and H-7'), 4.00 (dd, 1H, J= 11.6 Hz, 8 Hz, H-2'” or H-6'), 4.23-4.31 (m, 1H, H-7'), 5.65 (d, J=8 Hz, H-5), $7.58(\mathrm{~d}, J=8 \mathrm{~Hz}, \mathrm{H}-8) \mathrm{ppm} ;{ }^{13} \mathrm{C}-\mathrm{NMR}\left(\mathrm{CD}_{3} \mathrm{OD}, 100 \mathrm{MHz}\right): \delta=166.8,153.0,147.9,102.2,76.4,71.8$, 71.5, 66.9, 63.9, 63.8, 48.2 ppm; IR (neat): 3352, 3056, 2931, 2875, 1667, 1456, 1129, $1101 \mathrm{~cm}^{-1}$; HRMS (ESI) m/z: for $\mathrm{C}_{11} \mathrm{H}_{16} \mathrm{~N}_{2} \mathrm{O}_{6}[\mathrm{M}+\mathrm{Na}]^{+}$, Calcd. 295.0906, Found 295.0915.

Figure 7. Structure $7 \mathbf{b}$.

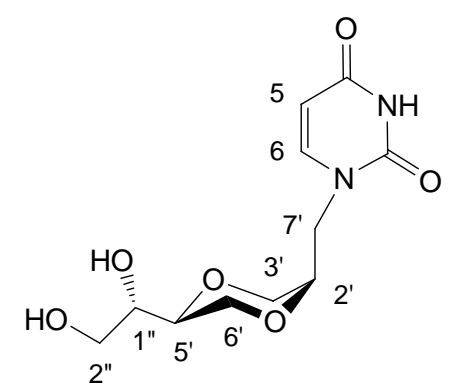

$7 b$ 
A mixture of adenine $(0.279 \mathrm{~g}, 2 \mathrm{mmol})$ and anhydrous potassium carbonate $(0.300 \mathrm{~g}, 2.2 \mathrm{mmol})$ in dry DMF $(10 \mathrm{~mL})$ was heated at $120^{\circ} \mathrm{C}$. After two hours, compound $4 \mathrm{a}(0.304 \mathrm{~g}, 0.9 \mathrm{mmol})$ was added to the solution. The mixture was stirred overnight at $120^{\circ} \mathrm{C}$. The mixture was concentrated under reduced pressure and the residue purified by flash chromatography using a mixture of dichloromethane and methanol (14:1) as the eluent. The product $(130 \mathrm{mg})$ was obtained in $42 \%$ yield. The product 11a exhibited the following spectroscopic properties: ${ }^{1} \mathrm{H}-\mathrm{NMR}\left(\mathrm{CDCl}_{3}, 400 \mathrm{MHz}\right): \delta=8.35(\mathrm{~s}, 1 \mathrm{H}, \mathrm{H}-2)$, 7.88 (s, 1H, H-8), 5.75 (s, 2H, NH ), 4.29 (dd, 1H, J=14.6 Hz, 3.4 Hz, H-7'), 4.12 (dd, 1H, J= 14.6 Hz, 6.8 Hz, H-7'), 4.01- 4.07 (m, 1H, H-4'), 3.97 (dd, J=11.4 Hz, 2.6 Hz, H-3'eq), 3.87-3.95 (m, 2H, H-2', H-5”), 3.75-3.82 (m, 2H, H-5”, H-6'), 3.46-3.57 (m, 2H, H-5', H-6'), 3.32 (dd, J= 11.4 Hz, 10.6 $\mathrm{Hz}, \mathrm{H}-3$ 'ax), 1.39 (s, 1H, H-10), 1.33 (s, 1H, H-10) ppm. ${ }^{13} \mathrm{C}-\mathrm{NMR}\left(\mathrm{CDCl}_{3}, 100 \mathrm{MHz}\right.$ ): $\delta=155.6$, 153.2, 150.4, 141.6, 141.1, 119.4, 109.7, 75.1, 74.9, 73.2, 68.5, 67.5, 65.0, 44.2, 26.2, 25.3 ppm; IR (neat): 3322, 3161, 2983, 2938, 2864, 1673, 1606, 1064, $1048 \mathrm{~cm}^{-1}$; HRMS (ESI) m/z: for $\mathrm{C}_{15} \mathrm{H}_{22} \mathrm{~N}_{5} \mathrm{O}_{4}$ $\mathrm{M}^{+}$, Calcd. 336.1671, Found 336.1676.

Figure 8. Structure 11a.

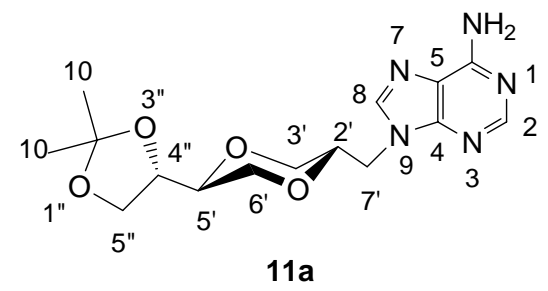

(2R,5S)-5-[(4S)-2,2-dimethyl-1,3-dioxolan-4-yl]-2-(adenin-9-yl-methyl)-1,4-dioxane (11b)

Synthesis of 11b was carried out as described for 11a. The product exhibited the following spectroscopic properties: ${ }^{1} \mathrm{H}-\mathrm{NMR}\left(\mathrm{CDCl}_{3}, 400 \mathrm{MHz}\right): \delta=8.35(\mathrm{~s}, 1 \mathrm{H}, \mathrm{H}-2), 7.87(\mathrm{~s}, 1 \mathrm{H}, \mathrm{H}-8), 6.14$ (s, 2H, $\mathrm{NH}_{2}$ ), 4.54 (dd, 1H, J=14.8 Hz, 8.8 Hz, H-7'), 4.32-4.38 (m, 2H, H-4', H-7'), 4.06- 4.11 (m, 1H, H-2'), 4.05 (dd, J=8.4 Hz, 6.6 Hz, H-5”), 3.80-3.90 (m, 3H, H-3', H-6'), 3.77 (dd, J=8.4 Hz, 6.8 Hz, H-5”), 3.61-3.67 (m, 2H, H-5', H-6'), 1.46 (s, 1H, H-10), 1.39 (s, 1H, H-10) ppm; ${ }^{13} \mathrm{C}-\mathrm{NMR}\left(\mathrm{CDCl}_{3}\right.$, $100 \mathrm{MHz}): \delta=155.7,153.1,150.1,140.9,119.4,109.9,74.6,73.8,71.1,65.6,65.1,63.0,42.8,26.5$, 25.4 ppm; IR (neat): 3276, 3134, 2984, 2935, 2870, 1676, 1600, 1575, 1126, $1066 \mathrm{~cm}^{-1}$; HRMS (ESI) $\mathrm{m} / \mathrm{z}$ : for $\mathrm{C}_{15} \mathrm{H}_{22} \mathrm{~N}_{5} \mathrm{O}_{4} \mathrm{M}^{+}$, Calcd. 336.1671, Found 336.1675.

Figure 9. Structure 11b.

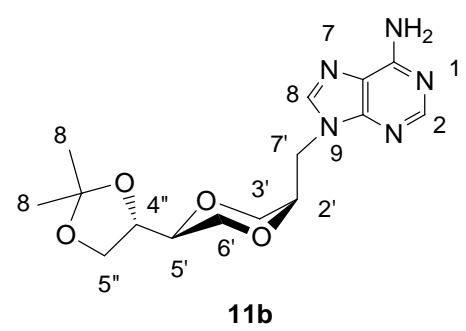


Compound 11a (108 mg, $0.32 \mathrm{mmol})$ was dissolved in methanol $(10 \mathrm{~mL})$, Amberlyst $15(45 \mathrm{mg})$ was added and the mixture was refluxed until TLC showed that all 11a was consumed. The solution was filtered and the solvent was evaporated. The product 12a $(81 \mathrm{mg}, 85 \%)$ exhibited the following spectroscopic properties: ${ }^{1} \mathrm{H}-\mathrm{NMR}\left(\mathrm{DMSO}_{6}, 400 \mathrm{MHz}\right): \delta=8.17(\mathrm{~s}, 1 \mathrm{H}, \mathrm{H}-2), 8.08(\mathrm{~s}, 1 \mathrm{H}, \mathrm{H}-8)$, 7.35 (brs, 2H, NH$)_{2}$ ) 4.3-4.8 (brs, 2H, OH), 4.21 (dd, 1H, J=14.4 Hz, $4.2 \mathrm{~Hz}, \mathrm{H}-7$ '), 4.13 (dd, 1H, J= 14.4 Hz, 6.8 Hz, H-7'), 3.83-3.88 (m, 2H, H-2', H-3'), 3.67-3.73 (m, 1H, H-6'), 3.17-3.48 (m, 6H, H6', H-5', H-3', H-1", H-2”) ppm; ${ }^{13}$ C-NMR (DMSO-d, $\left.100 \mathrm{MHz}\right): \delta=155.5,151.9,149.6,141.6$, 118.4, 75.2, 72.6, 70.8, 68.1, 67.5, 61.9, 43.7 ppm; IR (neat): 3271, 3117, 2918, 2881, 1668, 1604, 1120, 1106, $1066 \mathrm{~cm}^{-1}$; HRMS (ESI) m/z: for $\mathrm{C}_{12} \mathrm{H}_{17} \mathrm{~N}_{5} \mathrm{O}_{4}[\mathrm{M}+1]^{+}$, Calcd. 296.1358, Found 296.1356.

Figure 11. Structure 12a.

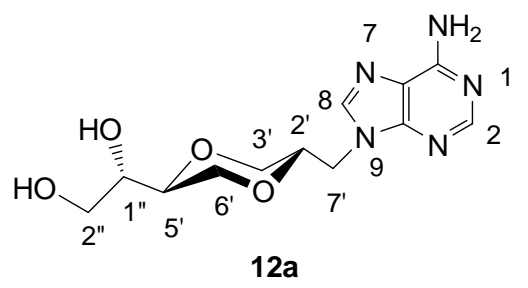

(2R,5S)-5-[(1S)-1,2-dihydroxyethyl]-2-(adenin-9-yl-methyl)-1,4-dioxane (12b)

Compound 12b was prepared using the same method as described for the synthesis of 12a. Thus, 11b (79 mg, $0.24 \mathrm{mmol})$ in methanol $(10 \mathrm{~mL})$ containing added Amberlyst-15 (36 mg) was refluxed until TLC showed that all 11b was consumed. The solution was filtered and the solvent was evaporated. The product 12b (49 mg, 70\%) exhibited the following spectroscopic properties: ${ }^{1} \mathrm{H}-\mathrm{NMR}$ (DMSO- $\left.d_{6}, 400 \mathrm{MHz}\right): \delta=8.16\left(\mathrm{~s}, 1 \mathrm{H}, \mathrm{H}-2\right.$ or H-8), 8.15 (s, 1H, H-8 or H-2), 7.30 (brs, $2 \mathrm{H}, \mathrm{NH}_{2}$ ), 4.69 (dd, 1H, J=14.4 Hz, 9.6 Hz, H-7'), 4.22 (dd, 1H, J=14.4 Hz, 4.2 Hz, H-7'), 4.00-4.05 (m, 1H, H2'), 3.92-3.98 (m, 1H, H-2” or H-6'), 3.78 (dd, 1H, J = 12.2 Hz, 2.2 Hz, H-3'), 3.69 (dd, 1H, J= 12.2 $\mathrm{Hz}, 3.2 \mathrm{~Hz}, \mathrm{H}-3$ '), 3.34-3.56 (m, 5H, remining protons) ppm; ${ }^{13} \mathrm{C}-\mathrm{NMR}$ (DMSO-d $6,100 \mathrm{MHz}$ ): $\delta=$ 155.7, 152.0, 149.6, 141.3, 118.5, 75.5, 70.4, 69.6, 65.6, 62.2, 61.4, 40.6 ppm; IR (neat): 3271, 3125, 2883, 1674, 1604, 1119, $1065 \mathrm{~cm}^{-1}$; HRMS (ESI) m/z: for $\mathrm{C}_{12} \mathrm{H}_{17} \mathrm{~N}_{5} \mathrm{O}_{4}[\mathrm{M}+1]^{+}$, Calcd. 296.1358, Found 296.1355.

Figure 10. Structure 12b.

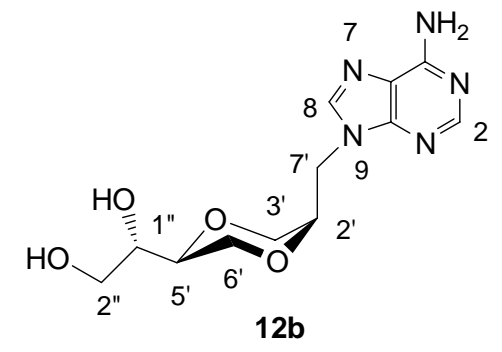




\section{Acknowledgements}

The authors wish to thank the Norwegian Research Council, NFR, for financial support. We will also thank Professor E. Uggerud, University of Oslo, for performing the mass spectrometric measurements.

\section{References and Notes}

1. Ichikawa, E.; Kato, K. Sugar-modified nucleosides in past 10 years, a review. Curr. Med. Chem. 2001, 8, 385-423.

2. Crimmins, M.T. New developments in the enantioselective synthesis of cyclopentyl carbocyclic nucleosides. Tetrahedron 1998, 54, 9229-9272.

3. Huryn, D. M.; Okabe, M. AIDS-driven nucleoside chemistry. Chem. Rev. 1992, 92, 1745-1768.

4. Hossain, N.; Blaton, N.; Peeters, O.; Rozenski, J.; Herdewijn, P.A. Synthesis of homo-Nnucleosides, a series of C1' branched-chain nucleosides. Tetrahedron 1996, 52, 5563-5578.

5. Hossain, N.; Hendrix, C.; Lescrinier, E.; Van Aerschot, A.; Busson, R.; De Clercq, E.; Herdewijn, P. Homo-N-nucleosides: incorporation into oligonucleotides and antiviral activity. Bioorg. Med. Chem. Lett. 1996, 6, 1465-1468.

6. Franzyk, H.; Rasmussen, J.H.; Mazzei, R.A.; Jensen, S.R. Synthesis of carbocyclic homo-N nucleosides from iridoids. Eur. J. Org. Chem. 1998, 12, 2931-2935.

7. Chun, M.W.; Kim, J.H.; Kim, M.J.; Kim, B.R.; Jeong, L.S. Synthesis of homo-N-nucleoside with 1,2,4-triazole-3-carboxamide. Nucleosides Nucleotides Nucleic Acid 2005, 24, 979-981.

8. Saladino, R.; Ciambecchini, U.; Hanessian, S. Synthesis of 1'-homo-N-nucleosides from hexitols. Eur. J. Org. Chem. 2003, 22, 4401-4405.

9. Yu, Q.; Kaffernik, S.; Carlsen, P. Synthesis of Optically Active 1,4-Dioxane Nucleotide Analogs. Synth. Commun. 2008, 38, 4250-4264.

10. Baskaran, S.; Aurich, H.G.; Biesemeier, F.; Harms, K. Cycloadducts from highly functionalized nitrones and oximes as ligands in the enantioselective addition of diethylzinc to benzaldehyde. $J$. Chem. Soc., PerkinTrans. 1 1998, 22, 3717-3724.

11. Nagashima, N.; Ohno, M. Selective monoalkylation of acyclic diols by means of dibutyltin oxide and fluoride salts. Chem. Pharm. Bull. 1991, 39, 1972-1982.

12. Nagashima, N.; Ohno, M. An efficient O-monoalkylation of dimethyl L-tartrate via O-stannylene acetal with alkyl halides in the presence of cesium fluoride. Chemistry Lett. 1987, 1, 141-144.

13. Marshall, J.A.; Andersen, N.H.; Hochstetler, A.R. Reduction of malonic enolates with lithium aluminum hydride J. Org. Chem., 1967, 32, 113-118.

14. Shi, M.; Jiang, J.-K.; Feng, Y-S. Chiral $C_{2}$-symmetric 2,3-disubstituted aziridine and 2,6disubstituted piperidine as chiral ligands in the addition reaction of diethylzinc with arylaldehydes. Tetrahedron Asymmetry 2000, 11, 4923-4933.

15. Marshall, J.A.; Beaudion, S. Stereoselective Synthesis of Higher Sugars by Homologation of Carbohydrate-Derived Enals with Nonracemic $\gamma$-(Silyloxy) Allylic Stannanes and SubstrateDirected Hydroxylation. J. Org. Chem. 1994, 59, 6614-6619. 
16. Albizati, K.F.; Babu, S.; Birchler, A.; Busse, J.K.; Fugett, M.; Grubbs, A.; Haddach, A.; Pagan, M.; Potts, B.; Remarchuk, T.; Rieger, D.; Rodrigues, R.; Shanley, J.; Szendroi, R.; Tobbetts, T.; Whitten, K.; Borer, B.C. A synthesis of the HIV-protease inhibitor nelfinavir from D-tartaric acid. Tetrahedron Lett. 2001, 42, 6481-6485.

17. Takano, S.; Kurotaki, A.; Sekiguchi, Y.; Satoh, S.; Hirama, M.; Ogasawara, K. Selective manipulation of hydroxy groups in (2S,3S)-threitol. Synthesis 1986, 10, 811-817.

18. Macritchie, J.A.; Peakman, T.M.; Silcock, A.; Willis, C.L. Iodocyclisation studies on unsaturated $\alpha$-Hydroxy esters. Tetrahedron Lett. 1998, 39, 7415-7418.

19. Bedford, S.B.; Bell, K.E.; Bennett, F. Model studies of the overall 5-endo-trig iodo-cyclization of homoallylic alcohols. J. Chem. Soc., Perkin Trans. 1 1999, 15, 2143-2153.

20. Allin, S.M.; Baird, R.D.; Lins, R.J. Synthetic applications of the amino-Cope rearrangement: enantioselective synthesis of some tetrahydropyrans. Tetrahedron Lett. 2002, 43, 4195-4197.

21. Greeves, N.; Lee, W.; Barkley, J. V. Synthetic applications (II) of the tandem [2,3]-Wittig-anionic oxy-Cope rearrangement: stereoselective trisubstituted-lactone and tetrahydropyran synthesis. Tetrahedron Lett. 1997, 38, 6453-6456.

22. Martinez, A.P.; Lee, W.W.; Improved synthesis of willardiine and 1-(2,2-diethoxyethyl)-uracil. $J$. Org. Chem. 1965, 30, 317-318.

23. Ahn, H.; Choi, T.H.; Castro, K.D. Synthesis and Evaluation of cis-1-[4-(Hydroxymethyl)-2cyclopenten-1-yl]-5-[ ${ }^{124}$ I] iodouracil: A New Potential PET Imaging Agent for HSV1-tk Expression. J. Med. Chem. 2007, 50, 6032-6038.

24. Joshi, R.V.; Zemlicka, J. Alkylation of adenine with tert-propargyl chlorides: acetylene / allene ratio and N9/N7 regioselectivity. Tetrahedron 1993, 49, 2353-2360.

25. Doel, M.T.; Jones, A.S.; Taylor, N. Approach to the synthesis of peptide analogs of oligonucleotides (nucleopeptides). Tetrahedron Lett. 1969, 27, 2285-2288.

Sample Availability: Selected samples are available from the authors.

(C) 2008 by the authors; licensee Molecular Diversity Preservation International, Basel, Switzerland. This article is an open-access article distributed under the terms and conditions of the Creative Commons Attribution license (http://creativecommons.org/licenses/by/3.0/). 